\title{
Postural Dysfunction During Standing and Walking in Children with Cerebral Palsy: What Are the Underlying Problems and What New Therapies Might Improve Balance?
}

\author{
Marjorie Hines Woollacott and Anne Shumway-Cook ${ }^{1}$ \\ Department of Human Physiology, University of Oregon, Eugene, Oregon; USA \\ ${ }^{1}$ Department of Rehabilitation Medicine, University of Washington, Seattle, Washington; USA
}

\begin{abstract}
In this review we explore studies related to constraints on balance and walking in children with cerebral palsy (CP) and the efficacy of training reactive balance (recovering from a slip induced by a platform displacement) in children with both spastic hemiplegic and diplegic CP. Children with CP show (a) crouched posture, contributing to decreased ability to recover balance (longer time/increased sway); (b) delayed responses in ankle muscles; (c) inappropriate muscle response sequencing; (d) increased coactivation of agonists/antagonists. Constraints on gait include (a) crouched gait; (b) increased co-activation of agonists/antagonists; (c) decreased muscle activation; (d) spasticity. The efficiency of balance recovery can be improved in children with $C P$, indicated by both a reduction in the total center of pressure path used during balance recovery and in the time to restabilize balance after training. Changes in muscle response characteristics contributing to improved recovery include reductions in time of contraction onset, improved muscle response organization, and reduced co-contraction of agonists/antagonists. Clinical implications include the suggestion that
\end{abstract}

Reprint requests to: M.H. Woollacott, Department of Human Physiology, University of Oregon, Eugene, OR 97403, USA improvement in the ability to recover balance is possible in school age children with $\mathrm{CP}$.

\section{REACTIVE STANCE BALANCE AND GAIT IN CHILDREN WITH CP}

Many children with cerebral palsy (CP) have poor walking abilities and manipulation skills. One contributing factor to their problems with gait and reaching movement is poor balance control because the maintenance of stability is critical to all movements. This review paper will summarize the research on reactive balance control in such children. Balance control is important for competence in the performance of most functional skills, helping a child to recover from unexpected balance disturbances, either due to slips and trips or to self-induced instability when making a movement that brings them toward the edge of their limit of stability.

Reactive balance control is defined as the ability of an individual to recover from an unexpected threat to balance, such as a slip or a trip. This characteristic is typically measured in the laboratory using a moveable forceplate on which the individual stands. The forceplate is unexpectedly moved different distances or speeds and individuals asked to respond to the threat without taking a step or reaching for a handrail unless they would otherwise lose balance. One behavioral measure of reactive balance control is the maximum velocity 
of platform movement at which a child can still maintain balance without taking a step or reaching for a handrail. Other measures include the time taken to recover stability or the total distance of center of pressure (COP, defined as the center of the vertical force exerted by the feet on the platform) movement during recovery from a platform displacement (Shumway-Cook \& Woollacott, 2000).

Research has shown that children with $\mathrm{CP}$ (spastic hemiplegia and diplegia) have to take a step at lower platform displacement velocities than typically developing children of the same age, take a longer time to recover stability, and show more center of pressure movement during the recovery period as well (Nashner et al., 1983; Chen \& Woollacott, 2003; Shumway-Cook et al., 2003). Figure 1 shows an example of the movement of the center of pressure from a child with spastic diplegia vs a child who is typically developing in response to the same amplitude and velocity of balance threat $(10 \mathrm{~cm}$ at $20 \mathrm{~cm} / \mathrm{sec})$. Note that the COP of the typically developing child passively moves forward as the platform moves backward. The child then efficiently returns the COP to a point close to its resting position as the child recovers stability.

In contrast, the child with spastic diplegia shows much greater COP movement before finally recovering stability, and in this case, is much farther than the typically developing child from the original resting point. This research shows that children with $\mathrm{CP}$ have more difficulty recovering balance efficiently when exposed to a balance threat. The next question to be asked is what neuromuscular constraints contribute to this reduced efficiency of balance recovery.

\section{COP Path for Forward Sway Perturbation}

Typically Developing Spastic Diplegia
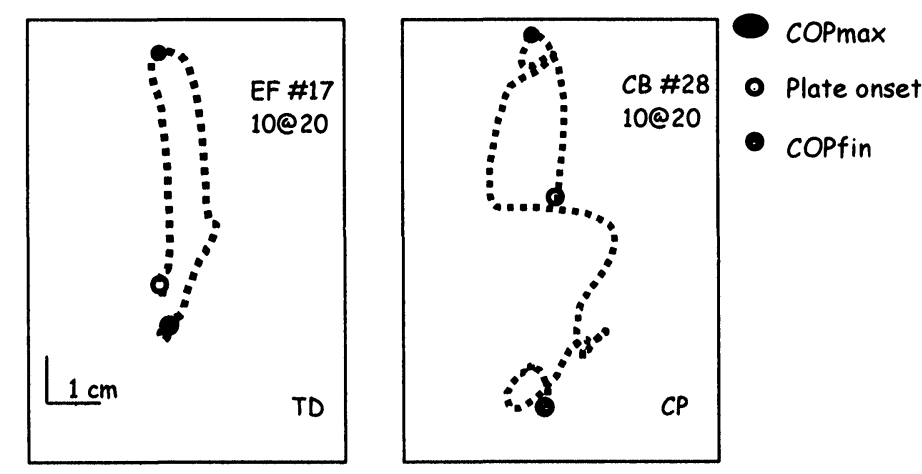

Fig. 1: Graph showing the total COP movement used to recover stability following a platform displacement causing forward sway for a typically developing child (left) and a child with spastic diplegia (right). Both children were exposed to similar types of perturbations 
Previous research by Nashner et al (1983) and Burtner et al (1998) has shown that the neuromuscular response characteristics that contribute to balance recovery show specific constraints in children with CP. For example, Nashner et al (1983) showed that children with spastic hemiplegia show problems with the timing of muscle responses. Such problems include delayed onset of contraction in the ankle musculature, a proximalto-distal muscle response sequence (hamstrings/ quadriceps muscles activated before gastrocnemius/ tibialis anterior) and co-contraction of agonist and antagonist muscles at a joint. Such changes in neuromuscular response characteristics would lead to a slower and less organized recovery of balance.

Burtner et al (1998) performed similar experiments comparing the postural responses of children with spastic diplegia of three developmental levels (pre-walkers, early walkers, experienced walkers) with those of typically developing children of similar developmental levels. The authors first noted that it was much more difficult to determine muscle response onsets and offsets for children with spastic diplegia because they typically have high levels of background muscle activity when quietly standing and long duration responses to balance threats as they attempted to recovery stability. Like Nashner et al (1983), the authors also found that timing of neuromuscular responses in these children was more typically disorganized (more proximal-distal activation and more co-contraction) and, unlike typically developing children who show improvement with developmental level, timing did not improve in children with spastic diplegia.

The investigators then asked whether one contributing factor to such poor neuromuscular response organization might be the crouched posture in which the children typically stood. To test this theory, they asked typically developing children of the same age to stand in a crouched posture and then measured their neuromuscular responses to platform induced slips. The authors found that in this position, the responses of the typically developing children showed (1) more coactivation of agonists/antagonists and (2) more proximal-to-distal muscle response timing. The results suggest that one contributing factor to disorganized muscle responses in these children is their musculoskeletal alignment leading to a crouched posture (Burtner et al 1998; Woollacott et al., 1998).

More recent work by Roncesvalles et al (2002) indicates that one contributing factor to the smaller limits of stability of children with spastic diplegia compared with typically developing children is a poor ability to increase muscle response amplitude when balance threats increase in magnitude. The group compared the muscle response amplitude (normalized and integrated EMG) for similar time periods after balance threats of increasing magnitude were imposed on typically developing children and on children with spastic diplegia. The authors found that typically developing children increase response size with level of difficulty of balance threat, whereas children with spastic diplegia are unable to do so.

Research on the gait characteristics of children with $\mathrm{CP}$ has shown similar results. Crenna and Inverno (1994) have defined the impairments in gait in children with $\mathrm{CP}$ as the following:

1. changes in mechanical properties of the muscle-tendon system (what they call the nonneural component) causing postural alignment problems and contributing to the crouch that also occurs in gait,

2. impaired muscle activation (what they define as the paretic component) that reduces muscle response output and contributes to fatigue, and

3. loss of selectivity in neuromuscular output (the co-contraction component). In addition, they found

4. abnormal velocity dependent EMG recruitment (the spasticity component) (Crenna, 1998). 
The neuromuscular constraints in both reactive balance and gait are so similar, suggesting that they have a common origin. In fact, researchers often note that gait is made up of both stability (not falling down) and progression (moving forward) requirements. Thus, it may be that many of the constraints on gait in children with $\mathrm{CP}$ are due to underlying stability problems, as noted above. In fact, the report of Crenna and Inverno (1994) shows more coactivation of agonist and antagonist muscles at the hip (quadriceps and hamstrings) when such children are performing unsupported walking (high balance requirements) than when performing supported walking (low balance requirements).

\section{REACTIVE BALANCE TRAINING IN CHILDREN WITH CP}

As research indicates that impaired balance shows a correlation with functional limitations in children with CP (McClenaghan et al., 1992; Crenna, 1998), therapy programs typically have an increase in postural function as one of their goals, with the aim of creating increased efficiency in both undisturbed stance and reactive balance control (Butler, 1998). Recently, research was performed to quantify the extent of improvement in balance with a specific type of balance training involving reactive balance control in a laboratory setting. The research was modeled after that in a previous study (Sveistrup \& Woollacott, 1997), which showed the effectiveness of intensive practice in reactive balance control on the organization of postural responses to balance threats in typically developing children just learning to stand independently.

These training studies (Shumway-Cook et al., 2003; Woollacott et al., in press) examined the effect of intensive practice in responding to balance threats created by a moveable platform on recovery of stability in school age children with CP (mean age $9 y, 4$ with spastic diplegia and 2 with spastic hemiplegia) (Shumway-Cook, et al., 2003). We hypothesized that this type of balance training would improve reactive balance control in children with $\mathrm{CP}$, specifically improving the performance variables that were found disorganized in prior research. Thus, we expected a reduction in both COP movements during balance recovery and in time taken to stabilize balance after a balance threat. We also expected that the improvement would be maintained for 1 month after the end of training.

Inclusion criteria for the study were the ability to stand independently for at least 30 seconds and to walk independently, either with or without an assistive device. Training involved five consecutive days of reactive balance training on a moveable platform. The training included 100 perturbations of about $4-6 / \mathrm{min}$ each (along with rest breaks), with platform movements in both anterior and posterior directions at different amplitudes (3-6 $\mathrm{cm})$ and speeds $(12-24 \mathrm{~cm} / \mathrm{sec})$. Each participant wore a safety harness and watched children's video during balance practice. The training differed from other intervention strategies in specifically focusing on reactive balance control during a short intensive period of training ( 5 days at 100 perturbations/day).

As hypothesized, balance training improved the ability of the children to recover efficiently from reactive balance threats. Figure 2 shows the COP traces for one of the trials in which a child with spastic diplegia recovered from a balance threat prior to training (a) immediately posttraining (b) and a month post-training (c). The figure shows a substantial improvement in the total movement of the COP during the platform perturbation and recovery during post-training and the one-month follow-up test, compared to pretraining (Shumway-Cook et al., 2003). In addition to a significant reduction in total COP movement during balance recovery, there was also a significant reduction in time to stabilize balance.

Figure 3 shows a plot of the mean time required to re-stabilize balance for different test 


\section{A: SD1 Pretest forward sway}

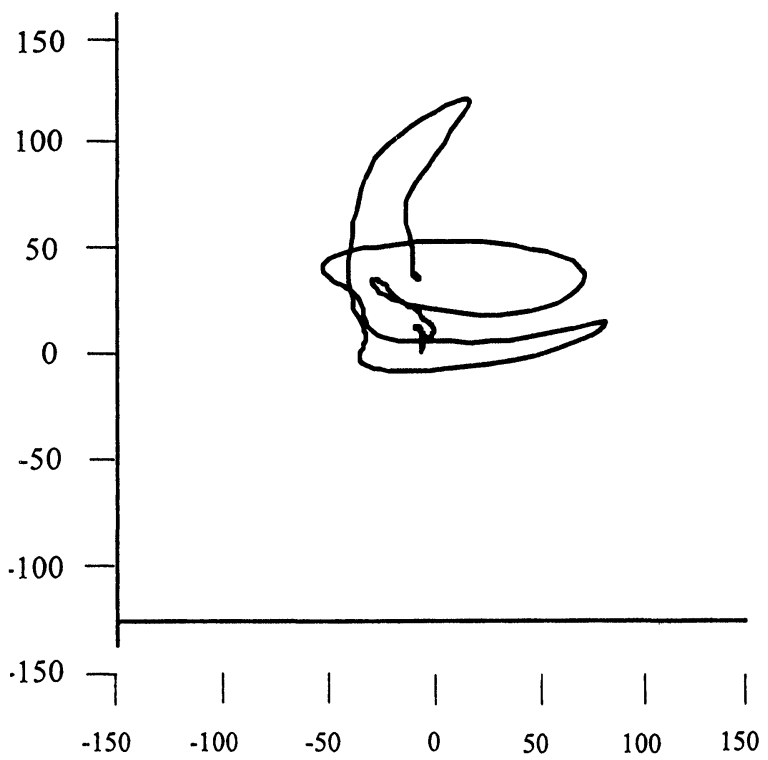

B: SD 1 Immediate Post Test Forward Sway

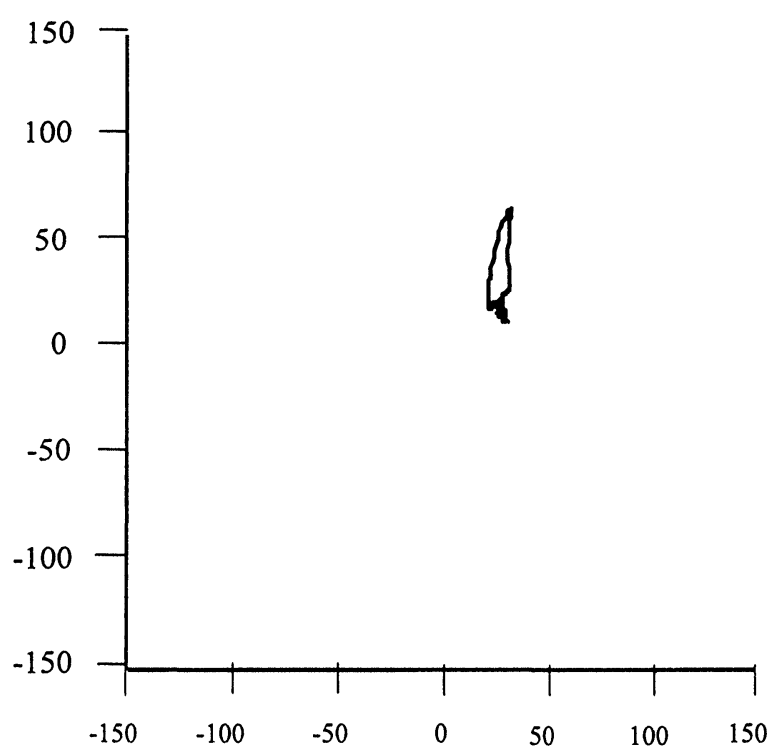

\section{C: SD 130 Day Post Test Forward Sway}

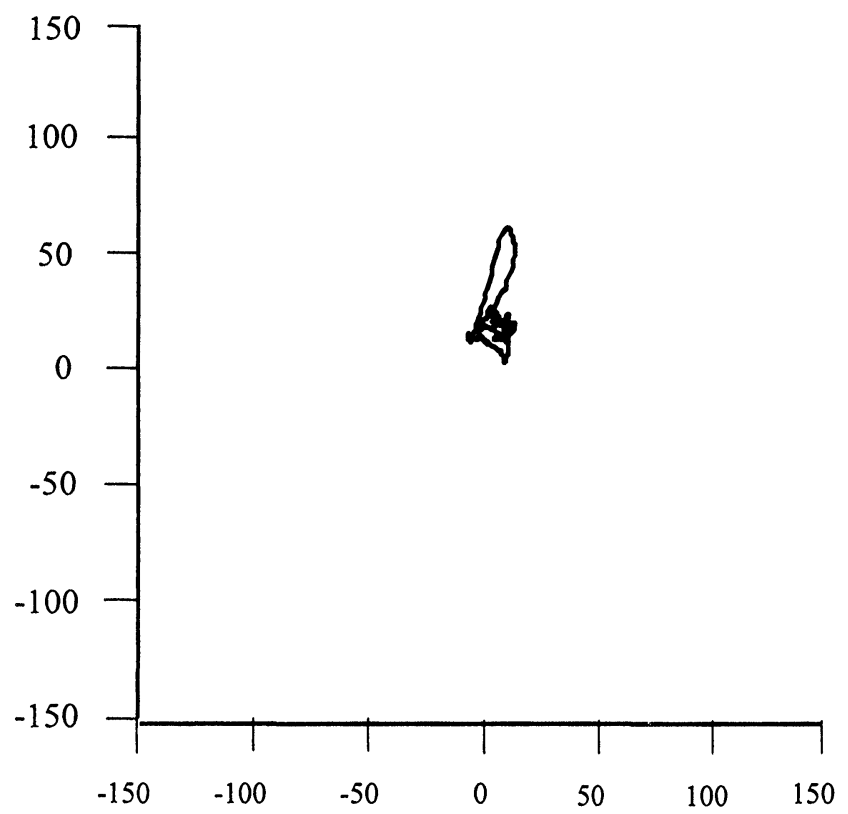

Fig. 2: Graph showing total COP movement from one pretraining (a), post-training (b), and one month post-training tests (c), in a child with spastic diplegia (SD1= child with spastic diplegia \#1. age $12 \mathrm{yrs}, 11 \mathrm{~m}$ ) (from Shumway-Cook et al., 2004). 
sessions (pre-training, during the intervention, and post-training) for each of the six children tested.

One question that is important to ask is whether improvement on a specific balance skill is associated with improvement on tests of functional balance, such as Dimension D of the Gross Motor Function Measure (GMFM) (Palisano et al., 1997). Five of six children were tested on the GMFM Dimension $\mathrm{D}$ before and after training. One child was unavailable after training. Although the GMFM does not include measures of reactive balance control, measuring only quiet stance and anticipatory (balance during voluntary movements) balance skills, three of five children showed improvement on the GMFM. One child with spastic hemiplegia and one child with diplegia improved their scores by $12 \%$ and $11 \%$, respectively. A second child with spastic diplegia showed a 3\% improvement.

To ascertain specific neuromuscular changes that were associated with improvement in reactive balance efficiency post-training, Woollacott et al. (in press) measured muscle response characteristics (surface electromyograms) both pre- and posttraining. The authors found the following improvements in neuromuscular response characteristics with training:

1. more efficient relative timing of contraction of distal and proximal muscles involved in the reactive postural muscle responses (distal muscles contracting before proximals).

2. shorter time to muscle contraction onset after training, contributing to faster balance recovery. and

3. reduced coactivation of agonist and antagonist muscles.

Figure 4 shows examples of changes in muscle contraction onset from pre-training to post-training and to 1 month follow-up tests. Note the reduction in onset of contraction for both platform displacements, causing both forward and backward sway.
Woollacott et al (in press) noted that different combinations of the above factors were used by each child, with more substantial improvement being found in the less involved children. For example, two children with spastic hemiplegia improved both contraction timing and reduced cocontraction of agonist/antagonist muscles after training. In contrast, only one of four children with spastic diplegia improved in both timing and level of co-contraction. Five out of six children reduced onset of contraction for ankle muscles for backward sway, contributing to a faster balance recovery. In addition, two children (one with spastic diplegia and one with hemiplegia) reduced the time of onset of contraction for distal muscles and lengthened it for proximal muscles, contributing to improvement in muscle response sequencing. One child with hemiplegia and two with diplegia showed later contraction times for antagonist muscles, reducing the coactivation of agonists with antagonists. Finally, one child with diplegia and two with hemiplegia showing smaller antagonist muscle contractions also reduced the coactivation of agonists and antagonists.

The wide variety of combinations of changes in neuromuscular response characteristics implies that each child find its own individual strategy for improving balance efficiency following training. The results also suggest that children with less involvement (hemiplegia vs diplegia) have more possibilities for increasing the efficiency of the available neuromuscular response characteristics.

The possible neural mechanisms underlying changes in neuromuscular response characteristics include the following:

1. improved proprioceptive sensitivity in leg muscles,

2. enhanced synaptic efficacy within primary sensorimotor cortex pathways, or

3. higher level adaptations at the level of cerebellum or association cortex. 


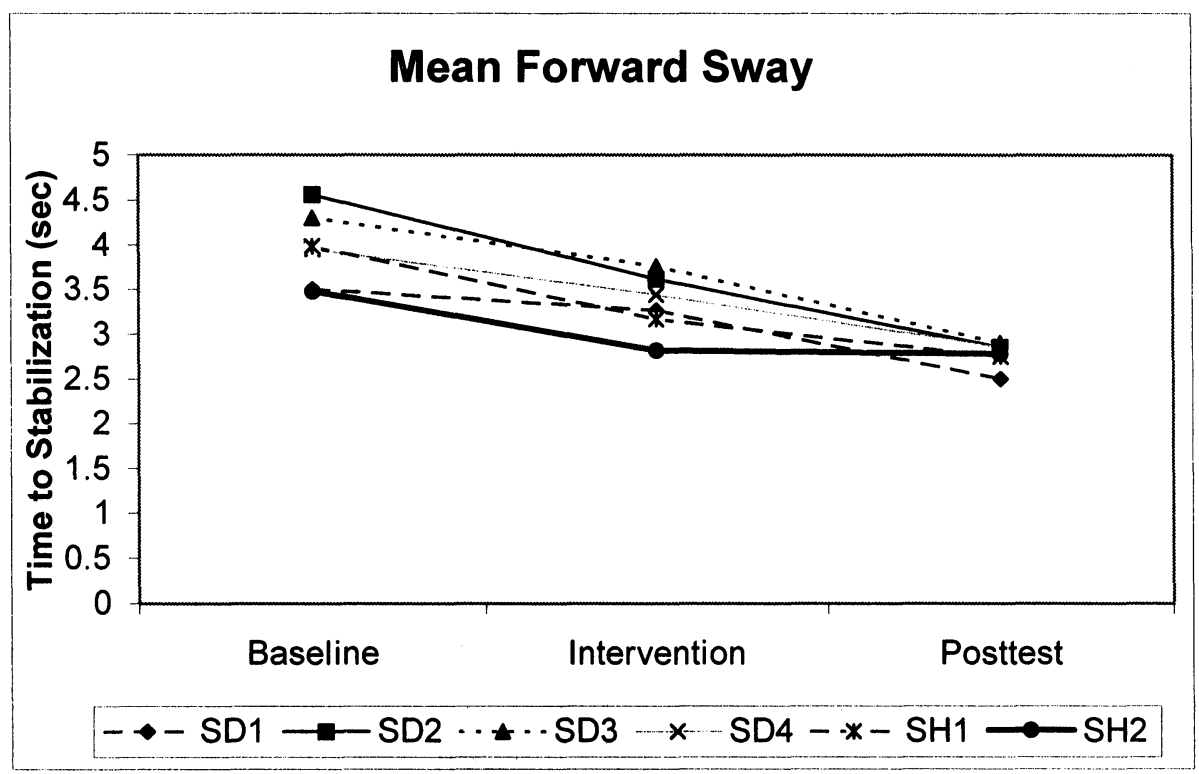

Fig. 3: COP time to stabilization (TTS) of balance after a forward sway perturbation in the six children, during baseline, intervention and maintenance (post-test) phases (Abbreviations: SD 1-4 = the 4 children with spastic diplegia. SH1-2 = the 2 children with spastic hemiplegia) (from Shumway-Cook et al., 2004).

\section{Children with Spastic Diplegia}

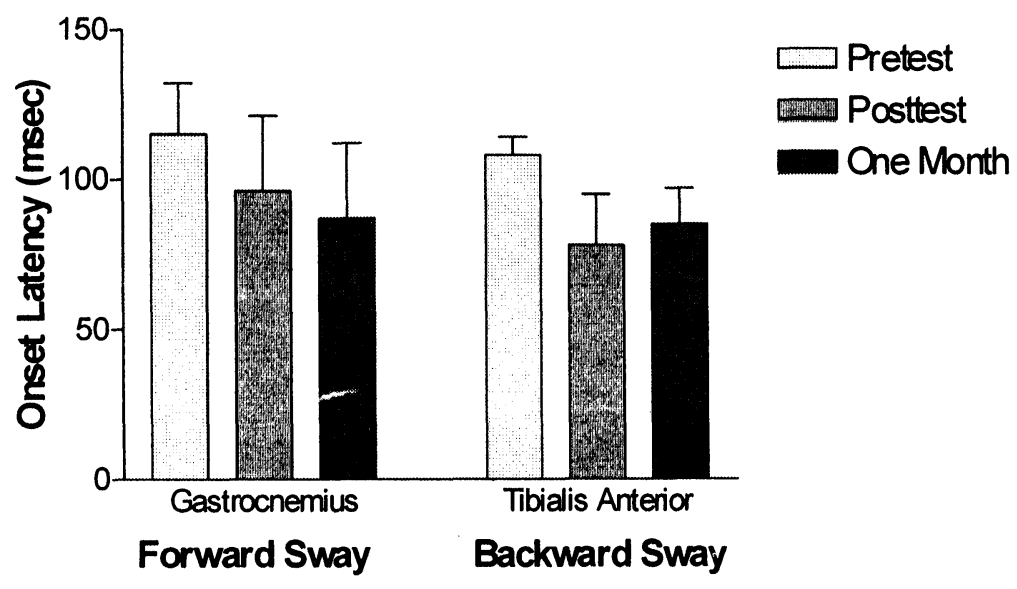

Fig. 4: Graph showing time of onset of contraction for the distal agonist muscles (gastrocnemius for forward sway and tibialis anterior for backward sway) for the children with spastic diplegia for pretest, posttest, and one month follow-up tests. 
Clinical applications of this study include the significant result that reactive balance performance can be improved in school age children with CP. The data suggest that continued practice in balance recovery and stability can help children with spastic hemiplegia and diplegia to improve their ability to maintain and recover balance.

\section{SUMMARY}

In summary, the results of the reported studies on reactive balance control in children with $\mathrm{CP}$ suggest that reactive postural control is reduced in children with both spastic hemiplegia and diplegia. This reduction includes a longer time to stabilize balance and more movement of the Center of Pressure during balance recovery. The neuromuscular response characteristics contributing to these balance constraints include a delayed onset of muscle contractions, a disorganized timing of muscle responses (proximal muscles activated before distal muscles), and an increased coactivation of antagonist muscles with agonists. The balance constraints also contribute to less-efficient walking performance, with research showing similar neuromuscular response changes in unsupported locomotion. Studies testing the efficacy of reactive balance training in school age children with $\mathrm{CP}$ have shown that balance can be significantly improved by as little as 5 days of intensive balance training (100 perturbations/day). In three of five children tested, changes found in the efficiency of reactive balance control, as measured by time to stabilize balance and mean COP displacement, were supported by the improvement on Dimension D of the GMFM. This subtest measures steady state and/or proactive balance control during stance. Currently, no test within the GMFM can measure reactive balance performance, thus, this parameter could not be measured directly in a functional balance test. The improvements were accompanied by both faster and better organized muscle contractions, including a reduction in the coactivation of agonist and antagonist muscles.

\section{ACKNOWLEDGEMENT}

This research was supported by NIH 1 R01Grant \#NS38714 to Dr. Marjorie Woollacott, PI.

\section{REFERENCES}

Burtner PA, Qualls C, Woollacott MH. 1998. Muscle activation characteristics of stance balance control in children with spastic cerebral palsy. Gait Posture 8: $163-174$.

Butler PB. 1998. A preliminary report on the effectiveness of trunk targeting in achieving independent sitting balance in children with cerebral palsy. Clin Rehabil 12: 281-293.

Chen J, Woollacott MH. 2003. Reactive balance in children with $\mathrm{CP}$ vs typically developing children: inverse dynamics. Society of Neuroscience Abstracts

Crenna P, Inverno M. 1994. Objective detection of pathophysiological factors contributing to gait disturbance in supraspinal lesions. In: Fedrizzi E, Avanzini G, Crenna P eds, Motor Development in Children. New York, NY, USA: John Libbey \& Co.; 103-118.

Crenna P. 1998. Spasticity and "spastic" gait in children with cerebral palsy. Neurosci Biobehav Rev 22: 571-578.

McClenaghan BA, Thombs L, Milner M. 1992. Effects of seat surface inclination on postural stability and function in the upper extremities of children with cerebral palsy. Dev Med Child Neurol 34: 40-48.

Nashner LM, Shumway-Cook A, Marin O. 1983. Stance Postural Control in Select Groups of Children with Cerebral Palsy: Deficits in Sensory Organization and Muscular Organization, Exp Brain Res 49: 393-409.

Palisano R, Rosenbaum P, Walter S, Russell D, Wood E, Galuppi B. 1997. Development and reliability of a system to classify gross motor function of children with cerebral palsy. Dev Med Child Neurol 39: 214-223. 
Roncesvalles N: Woollacott M: Burtner P. Neural factors underlying reduced postural adaptability in children with cerebral palsy: Neuroreport 2002. 13: 2407-2410.

Shumway-Cook A, Woollacott M. 2000. Motor Control: Theory and Practical Applications. Baltimore, Maryland, USA: Lippincott/Williams \& Wilkins.

Shumway-Cook A: Hutchinson S, Kartin D, Price R. Woollacott M. 2003. The effect of balance training on recovery of stability in children with cerebral palsy. Dev Med Child Neurol 45: 591-602.

Sveistrup H, Woollacott M. 1997. Practice modifies the developing automatic postural response. Exp Brain Res 114: 33-43.

Woollacott $\mathrm{MH}$, Burtner $\mathrm{P}$, Jensen J, Jasiewicz J, Roncesvalles R, Sveistrup H. 1998. Development of postural responses during standing in healthy children and children with spastic diplegia. Neurosci Biobehav Rev 22:583-589.

Woollacott M, Shumway-Cook A, Hutchinson S, Ciol M, Price R, Kartin D. In press. The effect of balance training on the organization of muscle activity used in the recovery of stability in children with cerebral palsy. Dev Med Child Neurol. 

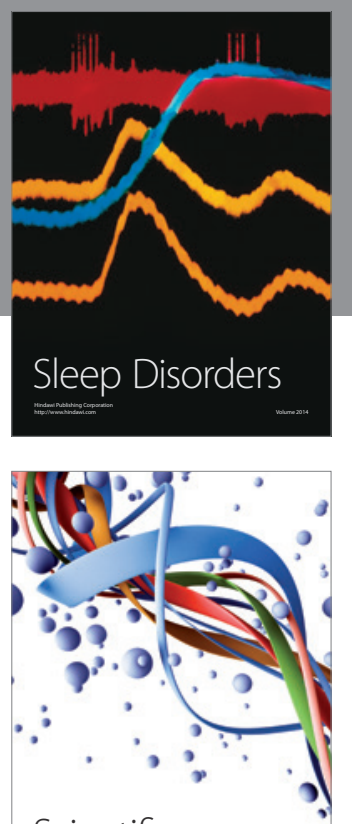

Scientifica
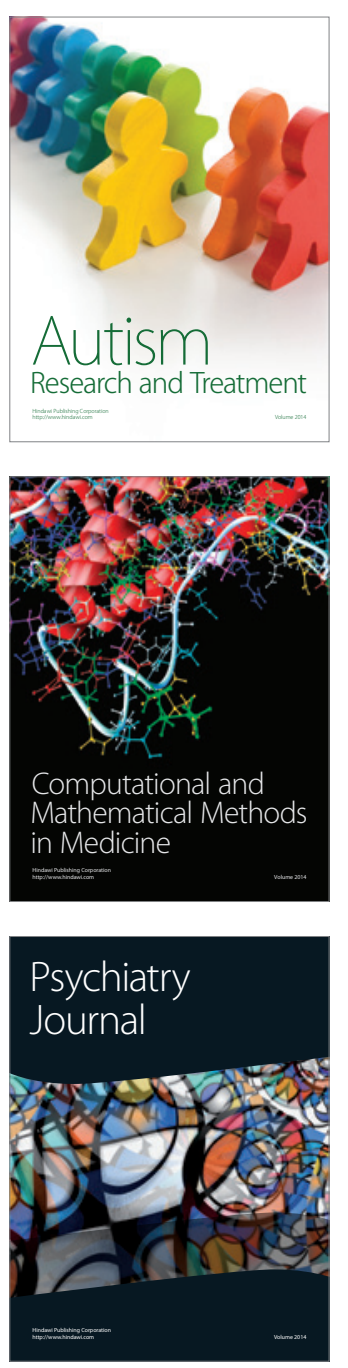
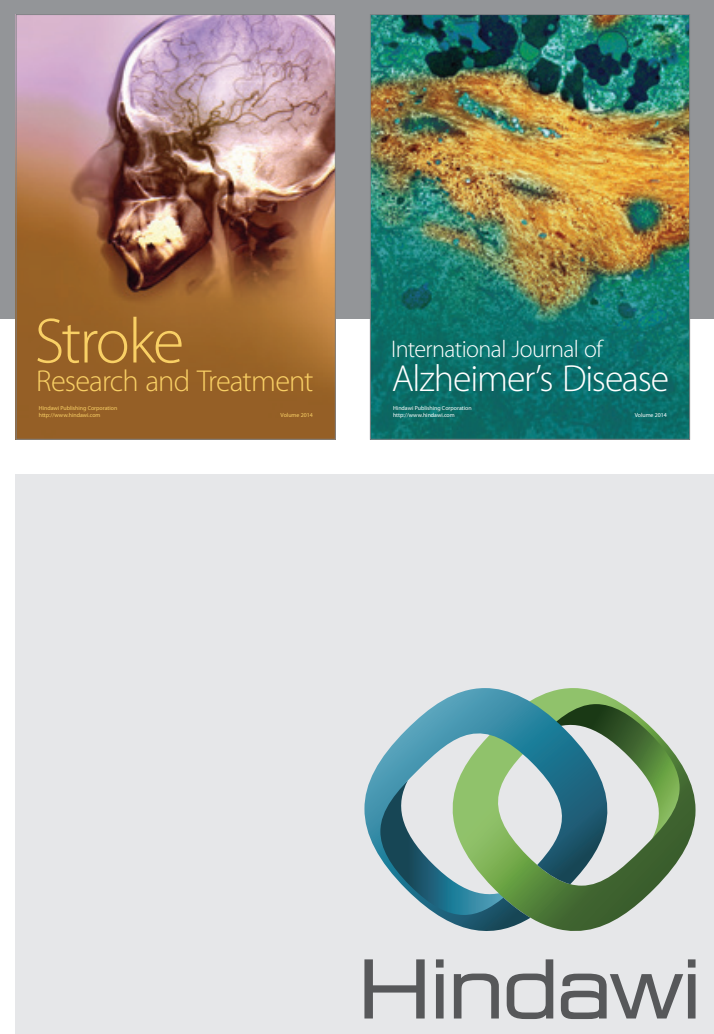

Submit your manuscripts at

http://www.hindawi.com
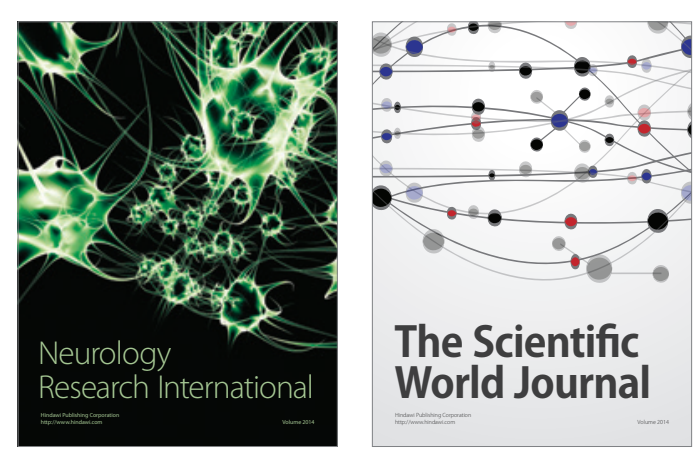

The Scientific World Journal

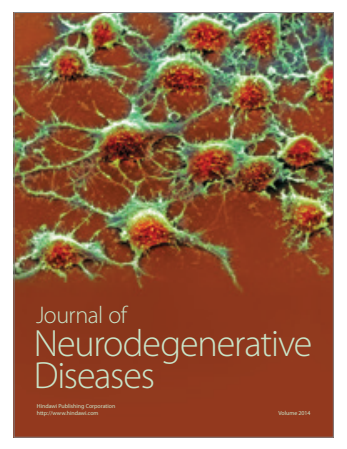

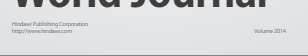

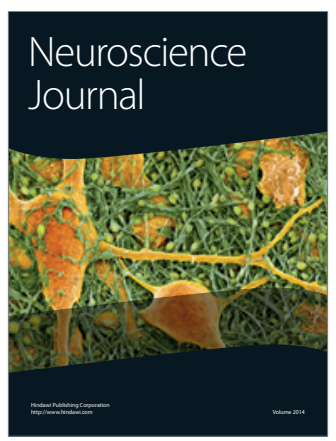

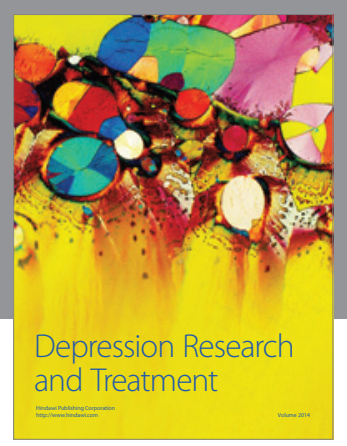
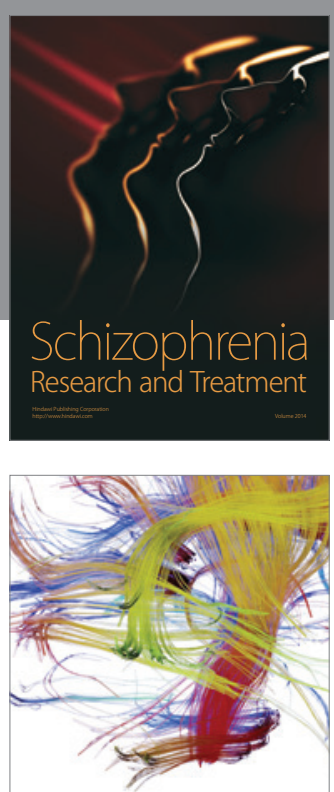

Brain Science

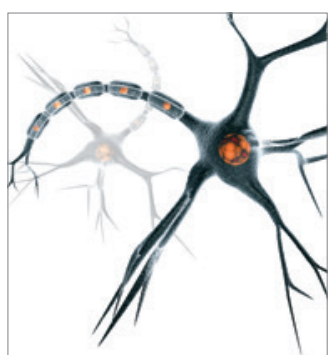

Neural Plasticity
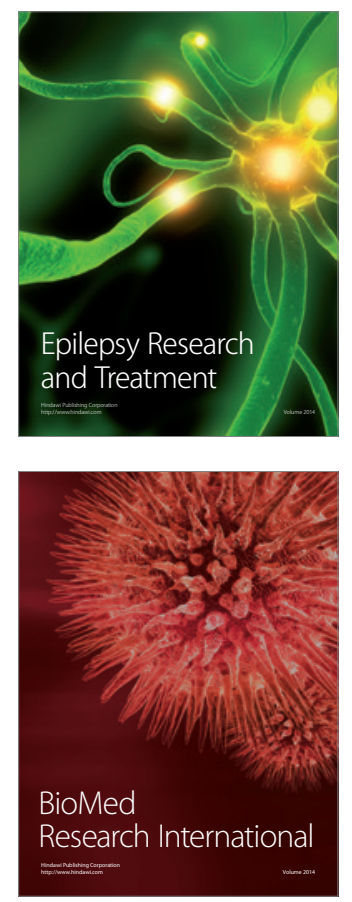

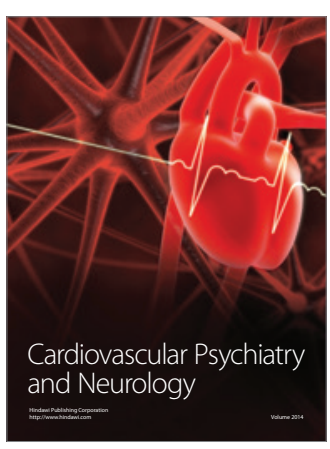

Parkinson's

Disease
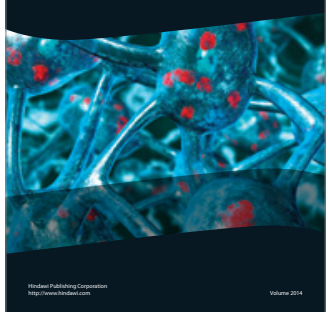